\title{
Psychological and emotional problems in staff following assaults by patients
}

\author{
AIMS AND METHOD \\ To determine psychological \\ morbidity among staff following \\ actual or threatened violence from \\ patients on acute psychiatric wards. \\ A retrospective survey of all 156 \\ clinical staff on Exeter's acute \\ psychiatric wards was conducted \\ by means of an anonymous \\ questionnaire using standardised \\ measures.
}

\author{
RESULTS \\ The response rate was $81 \%$, and $72 \%$ \\ of respondents had experienced \\ one or more distressing incidents of \\ actual or threatened violence within \\ the previous year. The 12-item \\ General Health Questionnaire and \\ Impact of Events Scale scores were \\ significantly higher in those who \\ reported exposure to 'frequent' \\ violent incidents.
}

\author{
CLINICAL IMPLICATIONS \\ Some National Health Service \\ staff may be harmed psychologically \\ when they are at work. This \\ consequently may have implications \\ for patient care, employer liability \\ and recruitment.
}

\section{Background}

Violence is increasingly prevalent in the UK. The Office for National Statistics in 1996 reported a doubling of notifiable violent offences recorded by police between 1984 and 1994. Health care workers are particularly vulnerable to violence at work (Health and Safety Advisory Committee, 1987; Whittington et al, 1996).

Within psychiatry, authors have studied the effects on staff of dealing with violent patients. A survey of post-traumatic stress disorder (PTSD) among clinical staff in two American psychiatric facilities (Caldwell, 1992) found that around $10 \%$ of respondents fulfilled a DSM-III-R (American Psychiatric Association, 1987) diagnosis of PTSD. In a recent study in Manchester by Guthrie et al (1999), psychiatrists of all grades found that dealing with violent patients was the most stressful aspect of their work. In Australia, a study of adverse experiences in psychiatric training found that assault by a patient, along with patient suicide, was identified as the most significant stressor (Kozlowska et al, 1997).

In 1998, the Royal College of Psychiatrists responded to increasing concern among its members regarding violent patients by publishing its clinical practice guidelines Management of Imminent Violence (Royal College of Psychiatrists, 1998). Standards drawn from these guidelines were then used in a national audit of the management of violence in mental health care settings conducted by the College Research Unit (Royal College of Psychiatrists, 2000).

\footnotetext{
Aims

Locally, staff concern about the perceived high levels of violence on acute psychiatric wards in Exeter provided additional impetus to conduct this survey. The aim was to determine levels of threatened or actual violence from patients directed towards staff working on acute psychiatric wards and to examine the psychological effects of such incidents.
}

\section{Method}

Subjects

All 156 clinical staff working on the acute psychiatric wards in Exeter were sent an anonymous questionnaire. Ward managers briefed staff in advance of receipt of the questionnaire in an effort to maximise response rates.

\section{Measures}

Staff were asked the question 'During the past 12 months, have you experienced any threats of, or actual, violence from patients on the wards that you found emotionally upsetting?'. The choice of responses was 'never', 'once or twice', 'several times' or 'frequently'.

Psychiatric caseness was estimated using the 12-item General Health Questionnaire (GHQ-12; Goldberg \& Williams, 1988), which is a widely used self-report measure designed for estimating minor psychiatric disorder in the general population. It is common practice to consider respondents scoring 3 or above as 'cases'. However, in common with Guthrie et al's study measuring psychological morbidity among psychiatrists in Manchester, we used a higher score of 4 or above as an estimate of caseness.

Post-traumatic symptoms in those staff experiencing distressing incidents of actual or threatened violence were determined by the Impact of Events Scale (IES; Horowitz et al, 1979). These symptoms were reported in relation to the most distressing incident experienced by the respondent during the previous year. Mean scores were compared and the subjects' total scores were classified according to the scheme recommended by the authors into 'low' (0-8), 'medium' (9-19) and 'high' (20+).

In addition to these standardised measures, subjects were questioned as to their gender, age, professional group and length of service on acute psychiatric wards. The questionnaire did not ask for actual descriptions of incidents or injuries sustained or attempt to make an objective classification of severity. Instead, respondents 
were asked to make a subjective assessment of the severity of the most distressing incident by rating how stressful they found the incident at the time using a choice of responses: 'mildly', 'moderately' or 'very stressful'. They were also asked whether or not they completed an incident form and took any time off work.

\section{Statistical analysis}

Analyses were performed using the Statistical Package for the Social Sciences (SPSS, version 8). For normally distributed data, means were compared using Student's t-test; $2 \times 2$ tables were analysed using odds ratios and confidence intervals.

\section{Results}

Of the 156 staff who were sent questionnaires, 127 $(81.4 \%)$ responded. Of the respondents, $62 \%$ were female and almost half were aged $25-40$ years; $13 \%$ were doctors, $76 \%$ were nurses and the remaining $11 \%$ were made up of occupational therapists, unspecified professions or those not responding to the question.

Ninety-one (72\%) of the respondents had experienced one or more incidents of actual or threatened violence from patients during the previous year that they had found distressing: 31\% of these found their worst incident 'mildly stressful' at the time, 37\% 'moderately stressful' and 32\% 'very stressful'. Nine (10\%) of these took time off work following their most distressing incident, with the majority $(7,78 \%)$ returning to work within a month. As noted previously, the questionnaire did not allow for descriptions of actual incidents or physical injuries sustained, but concentrated instead on the psychological impact of the incident as perceived by the staff member.

The mean GHQ score in the sample overall was 2.2, with $34(27 \%)$ of the respondents scoring 4 or more. There was a significant variation in GHQ-12 score with gender, the mean GHQ-12 score for men being 3.17 (95\% Cl=2.01-4.33) and for women being 1.67 (95\% $\mathrm{Cl}=0.51-2.83)$. No significant differences were found by age or professional group.

Taking the subgroup of respondents who reported experiencing one or more incidents of threatened or actual violence, nurses were much more likely than other professional groups to experience such incidents: odds ratio $(O R)=8.67(95 \% \mathrm{Cl}=3.01-25.6)$. There were no significant differences in terms of gender or age from the sample overall.

The subgroup of respondents experiencing incidents of actual or threatened violence were significantly more likely to score above the threshold on GHQ-12, indicating likely psychiatric caseness compared with the sample overall: $\mathrm{OR}=3.57$ (95\% $\mathrm{Cl}=1.06-13.18)$. The mean GHQ-12 score in the subgroup was 2.62, compared with 1.56 in the sample overall.

The mean IES score in the subgroup experiencing incidents was $15.55 \%$ of respondents scored within the low range and $37 \%$ within the high range. There were no values for the sample as a whole because this measure can be used only in relation to a specific incident. The mean IES score was significantly higher in men experiencing such incidents (21.7) than in women (10.91):

Student's $t$-test $=2.70 ; P=0.001$. There were no significant differences in terms of professional group.

Respondents were asked to estimate the frequency of incidents experienced over the previous year. Respondents experiencing several or more incidents had significantly higher mean GHQ-12 and IES scores than those who only experienced one or two incidents (see Table 1).

Only $60 \%$ of respondents experiencing incidents of actual or threatened violence that they found emotionally distressing completed an incident form. The questionnaire did not ask for reasons why forms were not completed. However, there were no significant differences between those completing or not completing an incident form in terms of gender, age, GHQ-12 score or IES score. Doctors were much less likely than other professional groups to fill in a form: $\mathrm{OR}=0.07(95 \% \mathrm{Cl}=0.00-0.65)$.

\section{Discussion}

Our study demonstrates that actual and threatened violence towards staff on acute psychiatric wards in Exeter is common. It is associated with significant psychological morbidity, particularly when of a repeated nature.

The main limitation of this study is recall bias. It is a retrospective, self-report survey that was anonymous in order to achieve a high response rate. It succeeded in this aim, but the design makes it impossible to distinguish between responders and non-responders.

Unlike many previous studies, we did not ask respondents to discriminate between actual or threatened violence or classify incidents in objective terms. This was because we were interested in the psychological effects of any incident causing subjective distress, however apparently 'minor' in terms of threat or actual injury sustained. Clearly the level of distress experienced in any particular situation will vary between individuals. We believe that the more open question used in our sample of clinical psychiatric staff makes any findings of psychological morbidity more relevant to other settings.

It is difficult to compare our findings with other studies because different rating scales and definitions of

Table 1. Frequency of incident and the GHQ-12 and IES scores

Frequency Mean GHQ-12 score Mean IES score of incident (confidence interval) (confidence interval)

'Once or twice' $1.33(0.58-2.08) \quad 10.57$ (5.9-15.24)

'Several times or $3.53(2.45-4.61) \quad 18.95$ (13.55-24.27)

frequently'

Significance $\quad P=0.002 \quad P=0.024$

GHQ-12, 12-item General Health Questionnaire; IES, Impact of Events Scale. 
violence and threatened violence were used. The incidence of violent or threatened violent episodes in our study was similar to that described by Caldwell in his study in two American psychiatric facilities. However, his definition was more stringent than ours, involving a serious threat to life or physical safety, or witnessing a serious injury or death. A total of $28 \%$ of Caldwell's respondents reported such an experience within the preceding 6 months. His survey used a diagnostic measure for PTSD and found that $61 \%$ of the sample overall reported symptoms of PTSD and that $10 \%$ fulfilled a diagnosis. The IES is a measure of the severity of post-traumatic symptoms and is not designed as a diagnostic tool. However, $37 \%$ of the respondents in our sample who experienced a violent or threatened violent incident scored within the 'high' range of the IES, which would indicate severe symptomatology.

The finding that only $60 \%$ of violent or threatened violent incidents are reported is encouragingly higher than in previous publications. Kidd \& Stark, in a survey in 1992, found that under $18 \%$ of junior doctors working in psychiatry reported assaults or threats. Lion et al reported a similar rate in 1981 among psychiatric staff in an American setting. There is no room for complacency, however, because official figures continue to underestimate the true extent of the problem.

There was a surprising gender difference in the GHQ-12 scores in the sample overall and in the IES scores, with men scoring significantly higher than women. This is in contrast to the tendency of women to suffer higher levels of psychiatric morbidity. It is not clear why this was the case.

Our study showed a significant association between increased numbers of incidents and higher scores on the GHQ-12 and IES measures. We decided not to ask respondents to quantify the numbers of incidents experienced in the past year because we felt that it would be difficult to recall exact numbers. Our finding of a significant association between higher numbers of incidents and higher scores on the GHQ-12 and IES measures gives rise to the possibility that earlier traumas make the emergence of PTSD following a subsequent incident more likely.

Violence and threatened violence to staff are common on acute psychiatric wards and have significant psychological effects. This surely must be an important recruitment and retention issue. Trusts may find themselves facing legal action in the future relating to industrial injury claims. Urgent action is needed to protect psychiatric staff in their work.

original

papers

\section{Declaration of interest}

None.

\section{Acknowledgements}

The authors would like to thank the staff of Wonford House Hospital for their participation in this survey, and Lin Winston and Val Wright for their invaluable secretarial support.

\section{References}

AMERICAN PSYCHIATRIC ASSOCIATION (1987) Diagnostic and Statistical Manual of Mental Disorders (3rd edn, revised) (DSM-III-R). Washington, DC: APA.

CALDWELL, M. (1992) Incidence of PTSD among staff victims of patient violence. Hospital and Community Psychiatry, 43, 838-839.

GOLDBERG, D. \& WILLIAMS, P. (1988) A Users Guide to the General Health Questionniare. Windsor: NFER-Nelson.

GUTHRIE, E., TATTAN,T., WILLIAMS, E., et al (1999) Sources of stress, psychological distress and burnout in psychiatrists. Psychiatric Bulletin, 23, 207-212.

HEALTH AND SAFETY ADVISORY COMMITTEE (1987) Violence to Staff in the Health Services. London: HMSO.

HOROWITZ, M., et al (1979) Impact of Event Scale: a measure of subjective stress. Psychosomatic Medicine, 41, 209-218.

KIDD, B. \& STARK, C. (1992) Violence and junior doctors working in psychiatry. Psychiatric Bulletin, 16, 144-145.

KOZLOWSKA, NUNN, K., COUSINS, P., et al (1997) Adverse experiences in psychiatric training. Part 2. Australian and New Zealand Journal of Psychiatry, $31,641-652$.

LION, J., SNYDER, W., MERRILL, G., et al (1981) Under-reporting of assaults on staff in a state hospital. Hospital and Community Psychiatry, 32, 497-498.

OFFICE FOR NATIONAL STATISTICS (1996) Notifiable offences recorded by the police; England and Wales. Annual Abstract of Statistics. London: Office for National Statistics.

ROYAL COLLEGE OF PSYCHIATRISTS (1998) Management of Imminent Violence (OP 41). London: Gaskell Publications.

- (2000) National Audit of the Management of Violence in Mental Health Settings: 1999-2000. London: Royal College of Psychiatrists.

WHITTINGTON, R. SHUTTLEWORTH, S., HILL, L., et al(1996) Violence to staffina general hospital setting. Journal of Advanced Nursing, 24, 326-333.

*Joanna Wildgoose Specialist Registrar in Psychiatry, Devon Partnership NHS Trust, Mental Health Services for Older People, Chadwell Centre,Torquay Road, Paignton, DevonTQ3 2DW, Martin Briscoe Consultant Psychiatrist, Devon Partnership NHS Trust, Keith Lloyd Senior Lecturer in Mental Health, Peninsula Medical School, Exeter 